\title{
THE DEVELOPMENT OF A COLLABORATIVE MEDICAL MODELLING SERVICE: ORGANISATIONAL AND TECHNICAL CONSIDERATIONS
}

Adrian Sugar BChD FDSRCS(Eng) FDSRCS(Ed) FDSRCPS(Glas) MD hc

Consultant Maxillofacial Surgeon, Morriston Hospital, Swansea NHS Trust, Morriston, Swansea SA6 6NL, Wales, UK

\section{Richard Bibb BSc (Hons) PhD AIMPT}

Research Fellow - Medical Application, The National Centre for Product Design and Development Research, University of Wales Institute, Cardiff, Western Avenue, Cardiff CF5 2YB, Wales, UK

Chris Morris HDCR DBA CI Cert

Radiology Services Manager, Morriston Hospital, Swansea NHS Trust, Morriston, Swansea SA6 6NL, Wales, UK

\section{John Parkhouse BSc (Hons)}

Formally Head of Information Technology and Telecommunications, Morriston Hospital, Swansea NHS Trust, Morriston, Swansea, SA6 6NL, Wales, UK

Corresponding Author:

Adrian Sugar

Consultant Maxillofacial Surgeon

Maxillofacial Unit

Morriston Hospital

Swansea SA6 6NL

Wales, UK

Tel: 01792-703077

Fax: 01792-703068

Email: adrian.sugar@swansea-tr.wales.nhs.uk 


\section{Summary}

The steps, problems, pitfalls and modifications made in the development of a collaborative medical modelling service for a general hospital in the UK are described. Emphasis is placed on the value of having as much control as possible in the hands of clinicians in order that the maximum relevant information can be obtained at minimum cost. 3D imaging, modelling and planning are now essential parts of any reconstructive surgery unit and must be adapted to make them as user friendly as possible for clinicians. 


\section{Introduction}

Medical Models are accurate physical replicas of human body parts created using Computer Tomography (CT) or Magnetic Resonance (MR) scan data together with a Rapid Prototyping (RP) process. ${ }^{1,2}$ Models made using RP methods are superior to milled models as the forming process is not inhibited by the shape of the object. ${ }^{3}$.

These models can be used to aid the treatment of patients. ${ }^{4,5,6}$ As an example, Figure 1 shows a model that has been used to plan a zygomatic arch construction in a patient with craniofacial microsomia. To date, most models have been of bone structures and have been used to aid reconstructive surgery but models can also be made of any body parts including soft tissue.

CT and MR scans are usually performed in slices or a spiral form in a plane perpendicular to the long axis of the patient. The results are displayed as a greyscale image for each slice scanned, or in the case of spiral-scans, a series of slices. In the case of CT scans the shades of grey shown relate directly to the density of the tissue. Values range from black representing air, to white representing cortical bone. In the case of MR scans the values relate to the magnetic resonance of the tissue. In general terms, CT is used for visualising skeletal structures whereas MR is better suited to visualising soft tissues.

The radiographer can isolate a particular tissue type by specifying upper and lower thresholds according to the greyscale value. This technique is referred to as segmentation. This is often used to view three-dimensional scans as a shaded image of 
the selected tissue (Figure 2). This segmented data can then be sent to the RP service provider where it can be used to generate the computer files required to build the physical medical model.

When a patient is scanned (for 3D scans) the interval between the slices may be in the order of 0.8 to 3 millimetres, whereas RP models are commonly built in layers 0.1 to 0.2 millimetres in thickness. Therefore additional software is used to interpolate intermediate slices between the scan data slices. Another interpolation is carried out within the plane of the scan to improve resolution. Together these operations result in an accurate 3D image with a natural appearance.

The interpolated data is then translated into an RP machine format, loaded into the machine and the model built. This will usually take several hours depending on the size and shape of the model and the RP process being used. The physical properties and accuracy vary according to process. For medical models the most commonly used RP processes are Stereolithography and Fused Deposition Modelling. All RP models can be handled, measured, drilled or sawn. This paper will not dwell on the technical aspects of $\mathrm{RP}$ processes as this is adequately described in other texts. 


\section{Aims of Medical Modelling Collaboration}

For most medical models produced in the UK, the preparation of the RP build files from patient scan data is undertaken by a very small number of specialist suppliers. One of the principal reasons for this is that scan data is only normally available in proprietary formats on archive media which cannot be read unless the appropriate hardware and software is purchased. This equipment differs for each manufacturer of medical scanner. Additional software is also required to segment the scan data and produce the RP build files. Many RP service providers are therefore unwilling to invest the large sums necessary to purchase this expensive software and equipment when the number of models made would make it difficult to recoup the investment.

Therefore in most cases scan data on archive media (e.g. magnetic optical disk, DAT tape or CD ROM) is posted from the radiology department to the specialist supplier. The supplier translates and segments the data from which the RP build files are created. This requires careful communication so that the correct parameters, tissues and extents are used. Once translation is complete the RP build files are then returned to the RP service provider who builds and delivers the model to the hospital.

This procedure, schematically shown in Figure 3 and called the "Disconnected" Procedure, is time consuming and removes potentially important clinical decision making opportunities from medical staff. 
In an effort to improve this situation, the authors piloted a more collaborative approach. Although the RP generation software needed to be purchased, the use of direct communication eliminated the need to translate data from the archive media saving a considerable amount of time and money. This process involved sharing the necessary procedures between the medical staff and the RP service provider. Effectively, the tasks were accomplished by the most appropriate staff, improving decision-making opportunities whilst reducing cost and turn around time. This approach was further accelerated by the use of electronic communication rather than routine mail.

Due to the modular nature of the software used, the Initial Integrated Procedure shown in Figure 4 was first attempted [7]. Experienced radiographers in a location accessible to the surgeons conducted the segmentation of the image data within the radiology department. The segmented data was then sent to the RP service provider who used the remaining software modules to prepare and manufacture the models. Once such a route was established, the turn around time from initial scan to finished model was reduced from weeks to days.

This approach had both technical and organisational implications as described in the next section.

\section{Implementation}

Several commercially available software packages enable the creation of physical models from medical scan data via computer-controlled processes such as rapid 
prototyping. However, the software used in this study consists of one module (Mimics ${ }^{\circledR}$, Materialise NV, Leuven, Belgium) used for the import and segmentation of scan data. A separate module (CT Modeller, $\mathrm{CTM}^{\circledR}$ ) generates the rapid prototyping build files from data exported from Mimics ${ }^{\circledR}$. This allowed the segmentation software (Mimics ${ }^{\circledR}$ ) to be installed in both the radiology and the clinical departments while the other module $\left(\mathrm{CTM}^{\circledR}\right)$ remained at the RP service provider. The segmentation module was installed on well-specified PCs connected to the hospital network allowing direct access to the scan data.

When using this software there is a convenient intermediate step between the different modules. The export file format from Mimics ${ }^{\circledR}$ to $\mathrm{CTM}^{\circledR}$ (called a .3dd file) is highly compressed, typically down to about $1 \mathrm{MB}$. Files this small are easily transferred as an attachment to an ordinary email. The use of existing email protocols including the firewalls at the hospital and the service provider eliminated security and network access issues.

After training and some initial trials, it became clear that file-import, preparation and segmentation was too time consuming to be undertaken by the radiographers. Although highly trained in the operation of the scanner, the radiographers were unfamiliar with other computer formats and network procedures. As the radiology department was already working at capacity, this was not the best use of their time. The simplicity of the software however allows accurate segmentation to be accomplished by any adequately 
trained user and is well within the competence of clinicians. Furthermore clinicians have a much better idea of what they want to achieve by the segmentation.

It was also felt that the majority of the clinical decision-making should be the responsibility of the clinical department. This would eliminate any potential misunderstandings between medical and technical staff. Therefore a modified procedure was implemented (Figure 5, The Current Integrated Procedure). This procedure involved the clinical department having the software to enable them to segment the data and email the compressed file format (a 3D image) to the RP service provider.

This method also keeps as much of the workload (and therefore cost) within the clinical department and minimises the workload transferred to radiology. The added benefit of this method is the capability of the clinical department to produce three-dimensional reconstructions on screen. The surgeon can then view and move these images at will rather than relying on a selection of fixed views produced on film by the radiology department. Axial slices can be viewed simultaneously with coronal and sagittal reformats and the slices can be run through in seconds. Areas of specific interest can be generated in three dimensions and viewed from any angle.

This increased access to two dimensional and three-dimensional data in various forms may also eliminate the need to produce a physical model at all in many cases, with a potential saving of time and money. The images produced can be saved on hard disk, printed on plain paper for storage in patients' notes and exported into standard image 
software and from there into slide presentation formats for teaching/lecturing/demonstrations. Hard copy can also be given to patients to help them understand their condition.

When following this procedure, the radiology department produces the scans in the usual manner and saves the data into a secure directory on a PC hard-wired to the scanner. This enables easy storage of data in the Radiology Department in their preferred format. The PC was connected to the hospital's local Intranet and through this makes the data available to the registered user in the Maxillofacial Unit using File Transfer Protocol (FTP) to another PC in the Maxillofacial Unit also connected to the Intranet. Other registered users in other hospital departments can also be included if necessary. The data is then burned in the Maxillofacial Unit onto CD, saved on the PC's hard disk and imported into the specialised imaging software (Mimics ${ }^{\circledR}$ ). Image manipulation can then be carried out by clinicians on screen in various planes as well as in 3D. Sections can be mirror-imaged and manipulated and areas measured without interfering with the schedule of a busy Radiology Department. The file for RP model construction, if required, can then be emailed to the RP provider as an attachment.

Of course when hospitals change their CT scanners, it is necessary to check that existing procedures still work and that the imaging software is configured appropriately to receive the new scan format. Current scanners almost invariably now comply to a standard digital format (DICOM) and for most good 3D software, the importation of such scans is actually made much easier. When Morriston Hospital acquired a new Multislice CT scanner in 2002, all its raw CT data was saved on a server within the 
hospital intranet. The Maxillofacial Unit was then given direct access to this server through the intranet and CT scans can be picked up directly and saved. This superceded the FTP arrangement.

\section{$\underline{\text { Discussion }}$}

\section{Technical Issues}

The most important technical issues in implementing such a modelling process are:

1. The export file format of the scan data

2. The nature of the storage media

3. The electronic transfer of the data

If the scan data media and/or format are proprietary, the appropriate software and hardware may have to be purchased by the collaborating parties. Many radiology departments are not accustomed to exporting data and may only have such archiving formats available as DAT or Optical Disk. In addition, some radiology departments are still not networked although this is likely to change over the next few years as teleradiology becomes more widespread. These data transport issues can be eliminated if the data can be directly transferred across computer networks. Electronic communication can be achieved in a number of ways and the method chosen should be economic and fast whilst maintaining security. 
Problems may be encountered when attempting to transfer very large data sets (a full set of 3D scan data may be 50-300 MB) as network disruptions become more probable during long download times. Such problems may result in corrupted or incomplete data. The use of compression software may help but the data sets may still be large. However evidence suggests that data is frequently transferred successfully in this manner. File Transfer Protocol (FTP) is commonly used for transferring data across local and remote networks. To maintain security FTP sites can be protected with user names and passwords as can access to servers.

Although the National Health Service (NHS) in the UK has a dedicated network (for example, in Wales the local network is called Digital All Wales Network or DAWN) it is, in the short term, unlikely that external RP service providers would be allowed access. Maintaining security is understandably a high priority for NHS networks. Even if access were allowed the network capacity is limited when compared to other high capacity networks, such as the Higher Education service 'JANET' (Joint Academic Network).

If the data files are small enough they can be sent as an email attachment. In this case security would be maintained through the email server's existing firewall. However, most email systems would become unsuitable if the file size exceeded $3 \mathrm{MB}$ or the number of files sent per day proves excessive, as most servers set storage limits for users. 
An ISDN line could be used but the security protocols for connecting to NHS networks may still apply in this case. If a PC is connected to a hospital Intranet (and through it the Internet), connection simultaneously to an ISDN line could breach firewall regulations. The installation of ISDN lines within the hospital is likely to require some form of management approval and agreement concerning which budget will cover the cost of installation, line rental and call charges. Once in place however, it should prove a reliable and secure transfer method.

Some consideration will have to be made concerning the amount of traffic the link will be expected to handle. It may well be that in the short term there will be insufficient traffic to warrant the investment in new networks. Alternatively, more commonly used high capacity storage systems such as CD-R, CD-RW, DAT, ZIP or JAZ drives could be used.

It is worth bearing in mind that the majority of medical models required will be for scheduled elective operations. In such cases, delay in communication of data can be anticipated and planned. However, in the long term, it is hoped that the service may be used to aid in the treatment of trauma victims in which case speed will be of great importance and a direct link may save crucial hours from the turn around time.

\section{Organisational Issues}

It is self-evident that setting up a successful dedicated medical modelling collaboration between a hospital and an RP service provider requires the full co-operation of the surgical departments, the radiology department, the IT department and the RP service 
provider. The implications for the different departments will be different depending on which technical solution is adopted. The chosen procedure should reflect the overall needs of the patient whilst taking due account of economic factors. Consequently the organisational considerations relate to:

\section{Economics and budgeting}

2. Staff workload and responsibility

Most hospital departments in the UK operate within strictly controlled budgets with limited resources. Obtaining the support and commitment of senior hospital management at the outset of a project such as this is therefore important. The costs involved in setting up the service will have to be met but there is likely to be pressure within departments to pass on as much of the cost to other sources of funding thus minimising the impact on departmental budgets. It is likely that clinical departments will want to maintain the maximum amount of responsibility and control whilst minimising workload and expenditure.

The use of medical models may drastically reduce theatre time and its associated costs as well as improving the quality and accuracy of surgery. However, cost savings have to be seen in context. For elective cases in the British National Health Service, the operating time available to surgeons is finite and spare time will be taken up by other cases. The principal effect, therefore, in a publicly funded healthcare system will be on waiting lists and waiting times for surgery. 
Quality of patient care and cost-effectiveness of care will improve but the need for a 3D model will impose an additional charge on tight budgets. Clinical control however of the process can enable many of these problems to be minimised. For example, Figure 6a shows a 3D CT reconstruction of a skull with a fronto-zygomatic bone defect. A model was required to enable a Cranioplasty plate to be constructed and to assist planning for the placement of craniofacial osseointegrated implants. By eliminating unwanted parts of the 3D image, a new 3D image (Figure 6b) was created by the clinicians. This was emailed in the form of a .3dd file to the RP provider. Being much smaller in size and volume, the model constructed was therefore very much cheaper. 


\section{Conclusions}

The clinical benefits of medical modelling have been documented and this work has demonstrated that a dedicated collaboration between the RP service provider and medical staff can greatly improve speed, efficiency and quality of service. Evidently, careful planning is necessary before embarking upon such collaboration. In particular, close co-operation between the rapid prototyping service provider, the Radiology department, Information Technology departments, surgical teams and hospital management is of the utmost importance. Although it is inevitable that investment will be required, all of the long-term clinical and economic benefits should be clearly indicated to fund managers. Besides the principal goal of creating a medical modelling service, the setting up of convenient data formats and electronic communications can be exploited in many other ways. This can be seen in the number of hospitals embracing tele-radiology which allows consultants to view medical images from remote locations.

It should be anticipated that existing computer facilities might have to be upgraded with particular attention paid to hardware specification, communication and data transfer issues. In some cases the scanner may also have to be networked or upgraded to allow the export of image data in a convenient format.

Although not in the form originally anticipated, the approach described here enabled a service to be established that provided the desired medical modelling service to the hospital in a fast, efficient and economic manner. The staff most able to complete each task effectively handles the individual procedures. Data transfer is fast and secure 
without compromising either party's network security. Importantly, the bulk of the costs and decisions are kept within the surgical department that requires the medical model and that department is thus able to control the process and minimise those costs.

In the near future it is likely that there will be many other factors driving hospitals to improve their networks and electronic communications. Issues regarding data format compatibility and file transfer will become more apparent and new standards applied to ensure the efficient and secure transfer of all kinds of clinical information. Many of the issues encountered in this collaboration will be overcome by the overriding proliferation of computerised clinical information in all hospitals. 


\section{$\underline{\text { Acknowledgements }}$}

This work has only been possible because of the enthusiasm and hard work of the entire collaborating team from PDR and Morriston Hospital. In particular the authors gratefully acknowledge the work of Peter Evans and Alan Bocca of the Maxillofacial Unit and Dr E Wyn Jones, Rosemary Davies and Sian Bowen of the Radiology Department of Morriston Hospital. We are also grateful to our Neurosurgical colleague, Tim Buxton, who has supported this project since its inception.

This project was supported by substantial grants from the Craniofacial Implant and Paediatric Craniofacial Surgery Endowment funds of Morriston Hospital and was also supported financially by both the hospital and university institutions. None of the authors have any pecuniary interest in the products mentioned. 


\section{$\underline{\text { References }}$}

1) Greenfield GB, Hubbard LB. Computers in Radiology. New York: Churchill Livingstone, 1984: 91 - 130.

2) Jacobs PF ed. Stereolithography and other RP\&M Technologies. Society of Manufacturing Engineering, One SME Drive, Dearborn, MI 48121, USA: 1996.

3) Klein HM, Schneider W, Alzen G, Voy ED, Gunther RW. Paediatric craniofacial surgery: comparison of milling and stereolithography for 3D model manufacturing. Pediatric Radiology 1992; 22: 458 - 460.

4) Bibb R, Freeman P, Brown R, Sugar A, Evans P, Bocca A. An investigation of three-dimensional scanning of human body parts and its use in the design and manufacture of prostheses. Journal of Engineering in Medicine 2000; 214 (H6): ISSN: 0954-4119, 589-594.

5) Bibb R, Brown R. The application of computer aided product development techniques in medical modelling. $37^{\text {th }}$ International ISA Biomedical Sciences Instrumentation, ISBN: 1-55617-722-4, 14-16 April 2000; 36: USAF Academy, Colorado Springs, USA, 319-324. 
6) Bibb R, Brown R, Williamson T, Sugar A, Evans P, Bocca A. The application of product development technologies in craniofacial reconstruction. Proceedings of the $9^{\text {th }}$ European Conference on Rapid Prototyping and Manufacturing, Athens, 17-19 July 2000: 113-122.

7) Swaelens B, Kruth JP. Medical applications of rapid prototyping techniques. Proceedings of the Fourth International Conference on Rapid Prototyping, Dayton, OH, USA, 1993: 107-120. 


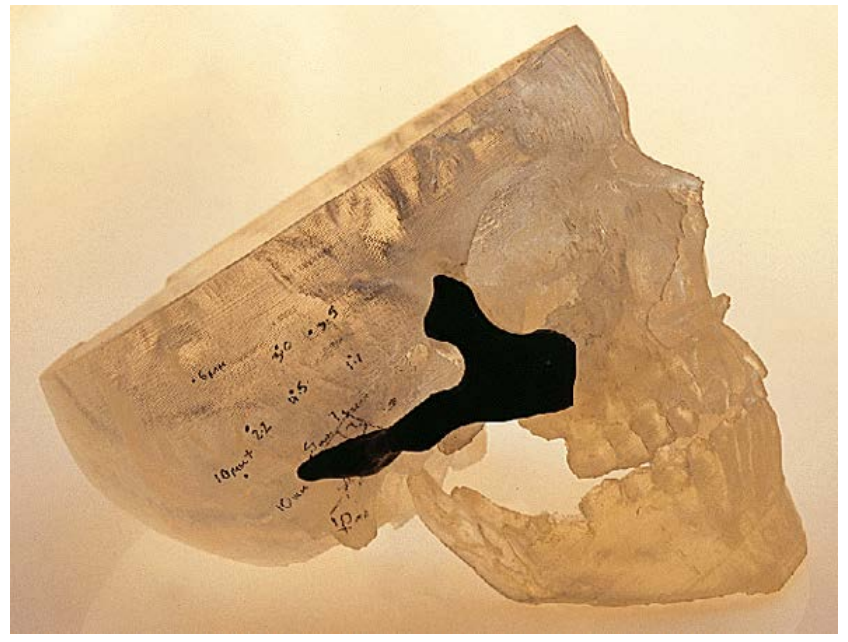

Figure 1. Medical Model of a skull made using stereolithography. 

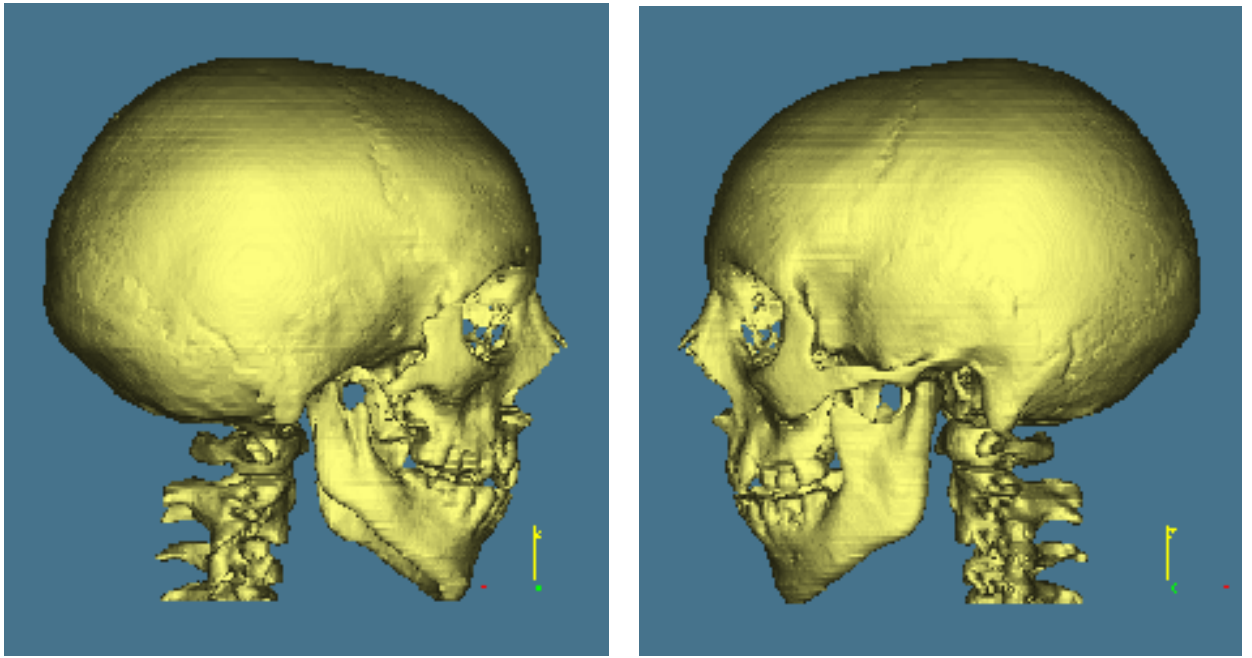

Figure 2. Typical 3D Reconstructions from CT Data: same case as in Figure 1. 


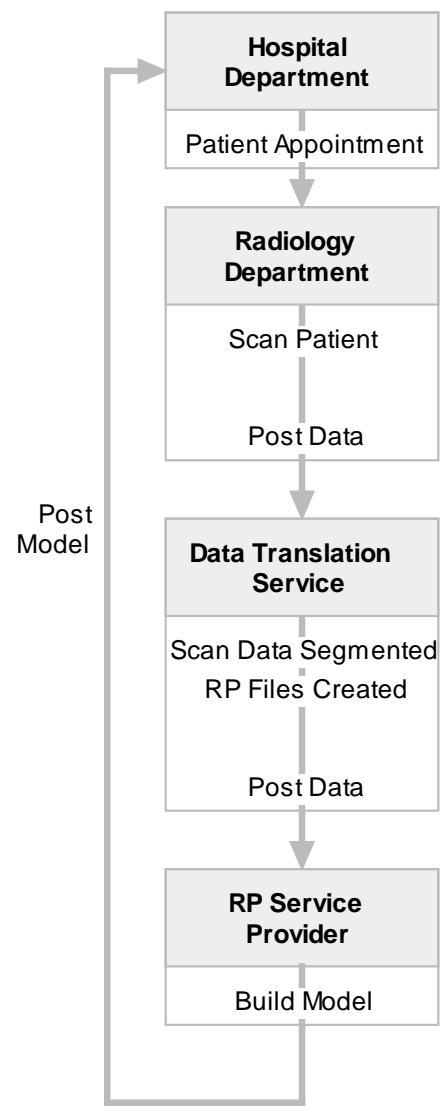

Figure 3. The 'Disconnected' Procedure 


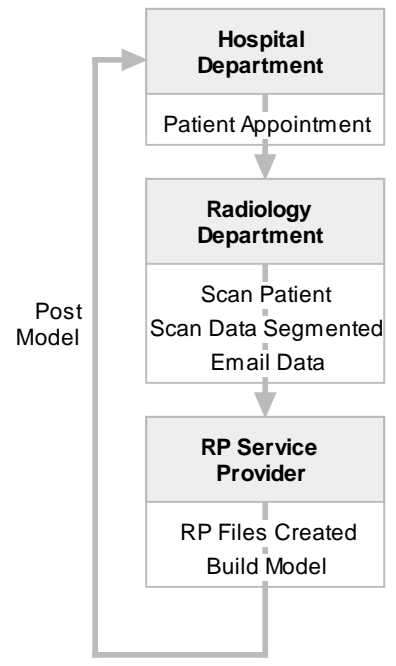

Figure 4. The Initial Integrated Procedure 


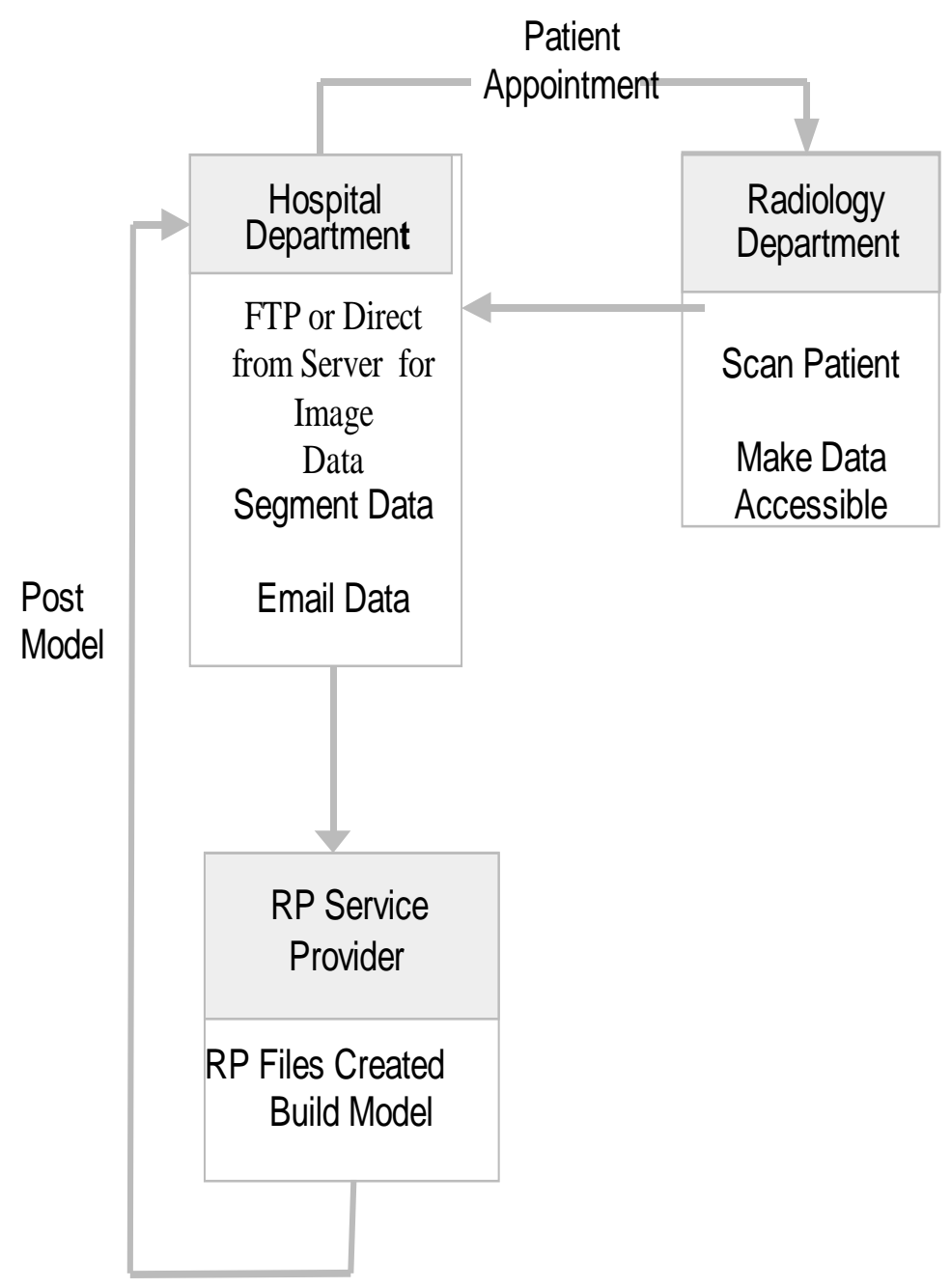

Figure 5. The Current Integrated Procedure 


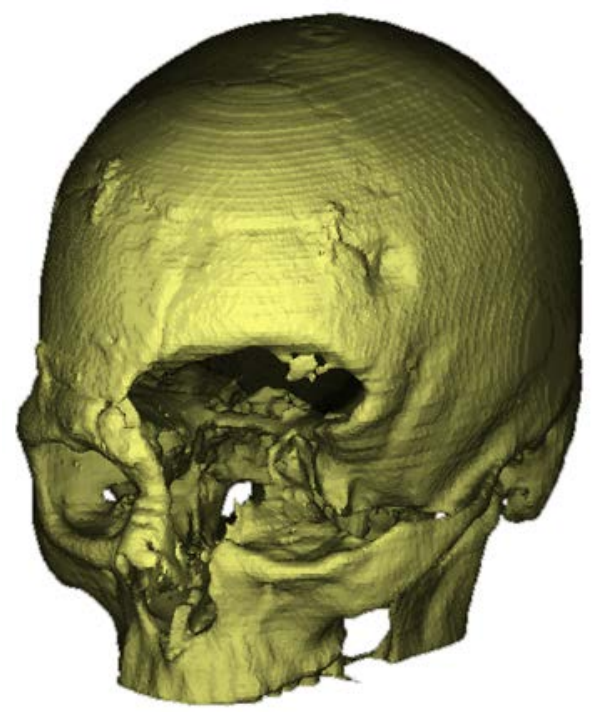

Figure 6a. Whole 3D Reconstruction

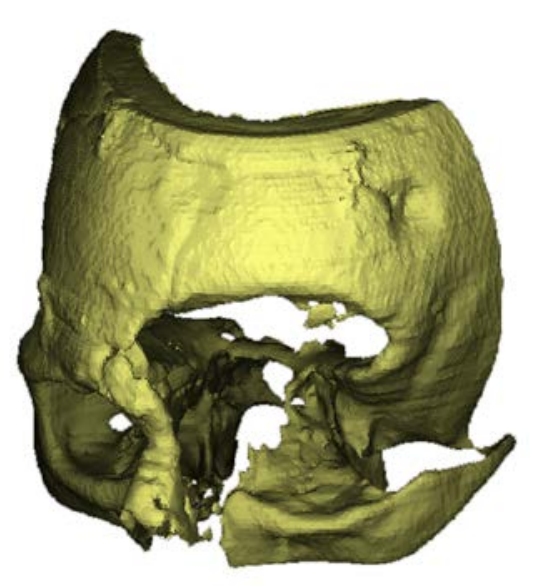

Figure 6b. Reduced to the area of interest 


\section{LEGENDS FOR FIGURES}

Figure 1. Medical Model of a skull made using stereolithography.

Figure 2. Typical 3D Reconstructions from CT Data: same case as in Figure 1.

Figure 3. The 'Disconnected' Procedure

Figure 4. The Initial Integrated Procedure

Figure 5. The Current Integrated Procedure

Figure 6a. Whole 3D Reconstruction

Figure 6b. Reduced to the area of interest 